\title{
Traveling waves for the Keller-Segel system with Fisher birth terms
}

\author{
GREGOIRE NADIN ${ }^{\dagger}$ \\ Département de Mathématiques et Applications, École Normale Supérieure, CNRS UMR 8553, \\ 45 rue d'Ulm, F 75230 Paris Cedex 05, France \\ BENOÎT PERTHAME \\ Université Pierre et Marie Curie-Paris 6, UMR 7598 LJLL, F-75005 Paris, France \\ and Institut Universitaire de France \\ LENYA RYZHIK ${ }^{\S}$ \\ Department of Mathematics, University of Chicago, Chicago, IL 60637, USA
}

[Received 29 June 2007 and in revised form 3 July 2008]

\begin{abstract}
We consider the traveling wave problem for the one-dimensional Keller-Segel system with a birth term of either a Fisher/KPP type or with a truncation for small population densities. We prove that there exists a solution under some stability conditions on the coefficients which enforce an upper bound on the solution and $\dot{H}^{1}(\mathbb{R})$ estimates. Solutions in the KPP case are built as a limit of traveling waves for the truncated birth rates (similar to ignition temperature in combustion theory).

We also discuss some general bounds and long time convergence for the solution of the Cauchy problem and in particular linear and nonlinear stability of the nonzero steady state.
\end{abstract}

2000 Mathematics Subject Classification: 35J60, 35K57, 92C17.

Keywords: Chemotaxis; traveling waves; Keller-Segel system; reaction-diffusion systems; nonlinear stability.

\section{The main result}

The growth of bacterial colonies undergoes complex biomechanical processes which underly the variety of interfaces exhibited by the colonies occupancy region. Usually cells divide and undergo active motion resulting in fronts of bacteria that are propagating and delimit a free boundary between the colonized and uncolonized areas. These fronts may be unstable leading to various patterns that have been studied for a long time, such as, for instance, spiral waves [17], aggregates [19] and dendrites [1, 11]. At least three elementary biophysical processes play commonly a central role in these patterns, and have been used in all modeling: (i) cell division which induces the growth of the colony, (ii) random cell motion-for instance, bacteria can swim in a liquid medium thanks to flagella, and (iii) chemoattraction through different molecules that the cells may release in their environment and that diffuse, leading to some kind of (possibly long distance) communication. Our purpose here is to study the existence of traveling waves and the linear and nonlinear stability of the

\footnotetext{
${ }^{\dagger}$ E-mail: gregoire.nadin@ens.fr

‡E-mail: benoit.perthame@upmc.fr

§E-mail: ryzhik@math.uchicago.edu
} 
steady states for a simple model combining these three effects. The macroscopic model describes the density of bacteria, denoted by $u(t, x)$ below, and the chemoattractant concentration $v(t, x)$ in the medium. It is a variant of the Keller-Segel system that has been widely studied in various contexts (see [5, 13, 20, 21, 7] and references therein).

We consider the one-dimensional Keller-Segel system with a Fisher-KPP birth term (we will refer to it as the Keller-Segel-Fisher system)

$$
\left\{\begin{array}{l}
u_{t}-u_{x x}+\chi\left(u v_{x}\right)_{x}=u(1-u), \\
-d v_{x x}+v=u
\end{array}\right.
$$

Here the notation $u_{t}$ or $u_{x}$ means time or space derivatives. The boundary conditions for $u$ and $v$ are

$$
v(-\infty)=u(-\infty)=1, \quad v(+\infty)=u(+\infty)=0,
$$

that is, there are no bacteria on the right. The two parameters $\chi$ and $d$ are, respectively, the sensitivity of the cells to chemoattraction, and the diffusion coefficient of the chemoattractant. The traveling wave solutions moving with a speed $c$ (which becomes a new unknown of the problem) for (1) are special solutions of the form $u(x-c t)$ and $v(x-c t)$ that satisfy

$$
\left\{\begin{array}{l}
-c u^{\prime}-u^{\prime \prime}+\chi\left(u v^{\prime}\right)^{\prime}=u(1-u) \\
-d v^{\prime \prime}+v=u
\end{array}\right.
$$

together with the boundary conditions (2). We prove the following result.

THEOREM 1.1 Let $\chi>0$ and $d>0$ satisfy

$$
\chi<\min (1, d) .
$$

Then there exists a traveling wave solution $\left(c_{*}, u, v\right)$ of 3 with the boundary conditions (2) and a constant $K(d, \chi)$ such that the functions $u(x)$ and $v(x)$ and the speed $c_{*}$ satisfy

$$
\begin{gathered}
0<u(x), v(x) \leqslant\left(1-\frac{\chi}{d}\right)^{-1}, \\
\int u(x)(1-u(x))^{2} \mathrm{~d} x+\int\left|u^{\prime}(x)\right|^{2} \mathrm{~d} x+\int\left|v^{\prime}(x)\right|^{2} \mathrm{~d} x \leqslant K(d, \chi), \\
2 \leqslant c_{*} \leqslant 2+\frac{\chi \sqrt{d}}{d-\chi} .
\end{gathered}
$$

Writing the second equation as a convolution $v=K_{d} \star u$, one may view this system as a Fisher equation with a nonlocal drift. Reaction-diffusion with nonlocal reaction or diffusion terms has recently been investigated (see [4, 8, 10, 12]), but, as far as we know, not for a nonlocal drift term. Nonlocal terms may make the homogeneous positive state unstable and then create periodic stable patterns. In this paper, we need some conditions on the coefficients, such as (4), that imply the stability of the state $u=v \equiv 1$.

Other situations where traveling waves appear in chemotaxis have been considered in the literature. For instance, [14] considers a source term for the chemoattractant in the equation on $v$, and [9] considers existence of traveling fronts by a linearization analysis (for small bacterial diffusion). There are also other related models of biological interest: see for instance the case of haptotaxis in [18]. We also refer to these papers for further references on fronts and waves for cell populations as well as to [22, 23] for the general theory of traveling waves. 
Our strategy for the proof of Theorem 1.1 is as follows. We introduce a smooth monotonic cut-off function $g_{0}(u)$ such that $g_{0}(u)=0$ for $u \leqslant 1$ and $g_{0}(u)=1$ for $u \geqslant 2$ and set $g(u)=$ $g_{0}\left(\left(u-\theta_{0}\right) / \theta_{0}\right)$-this function has a cut-off $\theta_{0} \in(0,1)$. Consider a regularized system

$$
\left\{\begin{array}{l}
-c u^{\prime}-u^{\prime \prime}+\chi\left(g(u) u v^{\prime}\right)^{\prime}=g(u) u(1-u) \\
-d v^{\prime \prime}+v=u
\end{array}\right.
$$

with the same boundary conditions (2). The system with the cut-off is of independent interest-the cut-off means that bacteria feel the chemoattractant and reproduce only if their density exceeds a critical threshold value. Mathematically, the role of the cut-off is very similar to that of the ignition temperature in combustion theory [15]. The first step in the proof of Theorem 1.1 is to construct a traveling wave solution $\left(c\left(\theta_{0}\right), u\left(x ; \theta_{0}\right), v\left(x ; \theta_{0}\right)\right)$ of $(8)$ for $\theta_{0}>0$ - as we have mentioned, this result is of independent interest. We do this for $\theta_{0}>0$ sufficiently small and also obtain (uniform in $\theta_{0}$ ) bounds on $c\left(\theta_{0}\right)$ and $u\left(x ; \theta_{0}\right), v\left(x ; \theta_{0}\right)$.

PROPOSITION 1.2 Let $\chi>0$ and $d>0$ satisfy

$$
\frac{1}{\chi}>\frac{1}{d}+1
$$

Then there exists $\alpha_{0}>0$ such that for all $\theta_{0} \in\left(0, \alpha_{0}\right)$ there exists a traveling wave solution $\left(c\left(\theta_{0}\right), u\left(x ; \theta_{0}\right), v\left(x ; \theta_{0}\right)\right)$ of $(8)$, 2). In addition, there exists a constant $K>0$ which does not depend on $\theta_{0}$ such that we have the following uniform bounds:

$$
\left\{\begin{array}{l}
0<u\left(x ; \theta_{0}\right), v\left(x ; \theta_{0}\right) \leqslant(1-\chi / d)^{-1}, \quad 0<1 / K \leqslant c\left(\theta_{0}\right) \leqslant K<\infty, \\
\int g\left(u\left(x ; \theta_{0}\right)\right) u\left(x ; \theta_{0}\right)\left(1-u\left(x ; \theta_{0}\right)\right)^{2} \mathrm{~d} x+\int\left|u^{\prime}\left(x ; \theta_{0}\right)\right|^{2} \mathrm{~d} x+\int\left|v^{\prime}\left(x ; \theta_{0}\right)\right|^{2} \mathrm{~d} x \leqslant K .
\end{array}\right.
$$

Here and throughout the paper we denote by $C$ and $K$ generic constants which may depend on $\chi$ and $d$ but not on the cut-off $\theta_{0}$ or the size $a$ of the approximating finite interval which appears later in the proof. We recall that in the case of a single equation with no chemoatractant coupling $(\chi=0)$ the speed $c\left(\theta_{0}\right)$ is unique for $\theta_{0}>0$ [15].

In this general framework it seems difficult to relax the size condition 9 , and to achieve the more general condition (4) that we use in Theorem 1.1. This is possible if we introduce two modifications in the above procedure. First, we consider another regularization of the system:

$$
\left\{\begin{array}{l}
-c u^{\prime}-u^{\prime \prime}+\chi\left(g(u) u v^{\prime}\right)^{\prime}=g(u) u(1-u) \\
-d v^{\prime \prime}+v=g(u) u
\end{array}\right.
$$

that is, the chemoattractant source also now has a small density cut-off. Second, we tune the truncation function appropriately - we now choose it with the following properties:

$$
\left\{\begin{array}{l}
g(u)=0 \quad \text { for } u \leqslant \theta_{0}, \quad g^{\prime} \geqslant 0, \quad g(u)=1 \quad \text { for } u \geqslant 1, \\
g(u)+u g^{\prime}(u) \leqslant 1+\alpha\left(\theta_{0}\right) \quad \text { with } \alpha\left(\theta_{0}\right) \underset{\theta_{0} \rightarrow 0}{\longrightarrow} 0, \\
g(u) \text { increases to } 1 \text { for } u \in(0,1) \text { as } \theta_{0} \rightarrow 0 .
\end{array}\right.
$$

The reader can easily check that these conditions are satisfied by the function

$$
g(u)=1+2 \alpha(1+\ln (u)-u)
$$

with $\alpha\left(\theta_{0}\right)$ normalized so that $g\left(\theta_{0}\right)=0$. 
Proposition 1.3 Assume that the cut-off $g$ has the properties (12) and that $\chi$ and $d$ satisfy the condition (4). Then there exists $\alpha_{0}>0$ such that for all $\theta_{0} \in\left(0, \alpha_{0}\right)$ there exists a traveling wave solution $\left(c\left(\theta_{0}\right), u\left(x ; \theta_{0}\right), v\left(x ; \theta_{0}\right)\right)$ of (11) with the boundary conditions 27 which satisfies the estimates $(5),(10)$ and

$$
K \leqslant c\left(\theta_{0}\right) \leqslant 2+(1+\alpha) \frac{\chi \sqrt{d}}{d-\chi},
$$

with a constant $K>0$ which does not depend on $\theta_{0} \in\left(0, \alpha_{0}\right)$.

This proposition allows us to pass to the limit $\theta_{0} \rightarrow 0$ and obtain a traveling wave solution of the original problem (3) without a cut-off as stated in Theorem 1.1 and with the smallness condition (4) on the chemotaxis. The traveling waves for a positive cut-off $\theta_{0}>0$ in Propositions 1.2 and 1.3 are constructed by first building an approximate solution on a finite interval $-a \leqslant x \leqslant a$ and then letting $a \rightarrow+\infty$, the strategy originated in [3].

This method enables us to find one speed $c\left(\theta_{0}\right)$ for a traveling wave. This is what we expect when there is a positive cut-off $\theta_{0}$ since there is a unique speed of propagation for the Fisher equation with ignition type nonlinearity. In the case of the Fisher equation with positive nonlinearity, it is well-known that there exists a half-line $\left[c_{*}, \infty\right)$ of speeds associated with traveling waves. The minimal speed gives the only stable wave, since the solution of the Cauchy problem with compactly supported initial data converges to this wave. In this paper we only prove that there exists at least one speed associated with a traveling wave for the Keller-Segel-Fisher equation. We do not know if this speed is minimal and if there exist any traveling waves with higher speeds.

By construction, the traveling wave solutions in Theorem 1.1 have a nonlinear stability property with respect to the perturbations of the birth term, under condition (4). This condition arises several times in our proof but we do not know if it is sharp: it implies the less restrictive condition $\chi<d$ which provides us with the maximum principle for $u$, but it is also instrumental in deriving the other fundamental a priori estimates in (10). It is interesting that the linear stability condition of the steady state solutions $(1,1)$ of (1) is much weaker than (4). To see that, we linearize the problem in the neighborhood of $(1,1)$ and write

$$
u=1+U, \quad v=1+V, \quad \text { where } U, V \ll 1 .
$$

One finds the linearized equations

$$
\left\{\begin{array}{l}
U_{t}-U_{x x}+\chi V_{x x}=-U \\
-d V_{x x}+V=U
\end{array}\right.
$$

Taking the Fourier transform we obtain

$$
\left\{\begin{array}{l}
\widehat{U}_{t}+k^{2} \widehat{U}-\chi k^{2} \widehat{V}=-\widehat{U}, \\
d k^{2} \widehat{V}+\widehat{V}=\widehat{U},
\end{array}\right.
$$

and since $\widehat{V}$ can be explicitly computed in terms of $\widehat{U}$, we reduce it to

$$
\widehat{U}_{t}+\left[k^{2}+1-\frac{\chi k^{2}}{1+d k^{2}}\right] \widehat{U}=0 .
$$

This equation is linearly stable if and only if

$$
k^{2}+1-\frac{\chi k^{2}}{1+d k^{2}} \geqslant 0 \quad \text { for all } k \in \mathbb{R} .
$$


Setting $X=k^{2} \geqslant 0$, we find the equivalent condition $1+X(d+1-\chi)+d X^{2} \geqslant 0$ for $X \geqslant 0$, which in turn is equivalent to

$$
\chi \leqslant(1+\sqrt{d})^{2} .
$$

In this case the steady state $(1,1)$ is linearly stable. When this condition is violated as in [9] unstable patterns arise. Condition (4) is of course stronger than (15) and even the sufficient condition $\chi<d$ for the uniform upper bound on $u$ and $v$ in (10) is still stronger than (15). This leaves open the question of the optimal condition for the existence of traveling waves.

The organization of this paper is as follows. We first consider the problem with cut-off on an interval $[-a, a]$ and prove the existence by a homotopy argument in Section 2 We also establish the main estimates in that section. In Section 3 we remove the cut-off and let the interval length $a$ tend to infinity, the main difficulty being to show that the states $(1,1)$ and $(0,0)$ are indeed connected by the solution obtained by this procedure. In the last section we establish some general bounds on the solution of the Cauchy problem and prove that the homogeneous solution is stable as soon as it is linearly stable.

\section{The problem on a finite interval $[-a, a]$}

Our approach follows the traditional methods (see [3] and [23], for instance), which we adapt to our specific situation. In particular, as usual, specific difficulties arise in showing that the speed $c$ is controlled from below and above, and that the states $u=1$ and $u=0$ are indeed reached at infinity (see [2, 6, 16] for an example where this question is left open in the construction of travelling waves for a reactive Boussinesq system).

\section{The finite interval approximation}

In order to prove Proposition 1.2, we first construct an approximation $\left(c_{a}, u_{a}, v_{a}\right)$ (we drop $\theta_{0}$ in the notation for the traveling wave for the moment) on a finite interval $-a \leqslant x \leqslant a$ :

$$
\left\{\begin{array}{l}
-c_{a} u_{a}^{\prime}-u_{a}^{\prime \prime}+\chi\left(g\left(u_{a}\right) u_{a} v_{a}^{\prime}\right)^{\prime}=g\left(u_{a}\right) u_{a}\left(1-u_{a}\right) \\
-d v_{a}^{\prime \prime}+v_{a}=u_{a}
\end{array}\right.
$$

The boundary conditions for $u_{a}$ are

$$
u_{a}(-a)=1, \quad u_{a}(a)=0 .
$$

Instead of imposing the boundary conditions for $v_{a}$ at $x= \pm a$, we extend $u_{a}$ to the whole real line as

$$
\bar{u}_{a}(x)= \begin{cases}1, & x<-a, \\ u_{a}(x), & -a \leqslant x \leqslant a, \\ 0, & x \geqslant a,\end{cases}
$$

and then we set

$$
v_{a}(x)=\int_{-\infty}^{\infty} K_{d}(|x-\xi|) \bar{u}_{a}(\xi) \mathrm{d} \xi, \quad K_{d}(\xi)=\frac{e^{-|\xi| / \sqrt{d}}}{2 \sqrt{d}}, \quad \int K_{d}(\xi) \mathrm{d} \xi=1 .
$$


The function $v_{a}(x)$ is defined for all $x \in \mathbb{R}$ and satisfies

$$
-d v_{a}^{\prime \prime}+v_{a}=\bar{u}_{a}, \quad v_{a}(-\infty)=1, v_{a}(+\infty)=0 .
$$

Three consequences of the representation formula $(19)$ are the bounds

$$
\begin{aligned}
& \left|v_{a}(x)\right| \leqslant\left\|u_{a}\right\|_{\infty}, \\
& \left|v_{a}^{\prime}(x)\right|=\frac{1}{2 d}\left|\int e^{-|x-\xi| / \sqrt{d}} \operatorname{sgn}(\xi-x) u_{a}(\xi) \mathrm{d} \xi\right| \leqslant \frac{1}{\sqrt{d}}\left\|u_{a}\right\|_{\infty}, \\
& \left|v_{a}^{\prime \prime}(x)\right| \leqslant \frac{C}{d}\left\|u_{a}\right\|_{\infty},
\end{aligned}
$$

which we frequently use.

In order to ensure that the solution $u_{a}$ has a nontrivial limit as $a \rightarrow+\infty$, we normalize it so that

$$
\max _{x \geqslant 0} u_{a}(x)=\theta_{0}
$$

This constraint indirectly fixes the speed $c_{a}$. It follows from the maximum principle and 22) that $u_{a}(0)=\theta_{0}$ and thus $u_{a}$ satisfies the boundary value problem on $[0, a]$ :

$$
-c_{a} u_{a}^{\prime}(x)-u_{a}^{\prime \prime}(x)=0, \quad 0 \leqslant x \leqslant a, \quad u_{a}(0)=\theta_{0}, u_{a}(a)=0 .
$$

Proposition 2.1 With the assumption (9), there exists a solution $\left(c_{a}, u_{a}, v_{a}\right)$ of (16), (17), (19), (22) with nonnegative functions $u_{a}$ and $v_{a}$, which in addition satisfies the uniform bounds [10].

The rest of this section is devoted to the proof of this proposition, which uses a homotopy argument. Accordingly, we introduce the homotopy parameter $\tau \in[0,1]$ and consider a family of problems

$$
\left\{\begin{array}{l}
-c_{\tau, a} u_{\tau, a}^{\prime}-u_{\tau, a}^{\prime \prime}+\chi \tau\left(g\left(u_{\tau, a}\right) u_{\tau, a} v_{\tau, a}^{\prime}\right)^{\prime}=\tau g\left(u_{\tau, a}\right) u_{\tau, a}\left(1-u_{\tau, a}\right), \\
-d v_{\tau, a}^{\prime \prime}+v_{\tau, a}=\tau u_{\tau, a},
\end{array}\right.
$$

together with the boundary conditions $(17)$, the first relation 19 (with the right side multiplied by the factor $\tau$ ) and normalization (22). To simplify the notation we drop the subscript $\tau$ below.

\section{A uniform upper bound for the traveling speed}

We begin with an upper bound for the speed.

LEMMA 2.2 If $d>\chi$, then any solution of (17), (19, , 22, , 23) satisfies

$$
0 \leqslant u_{a}(x), v_{a}(x) \leqslant(1-\chi / d)^{-1}, \quad\left|v_{a}^{\prime}(x)\right| \leqslant C,
$$

with the constant $C>0$ which depends only on $d$ and $\chi$. In addition, there exists a constant $a_{0}\left(\theta_{0}\right)>0$, and a constant $K>0$ which depends only on $d$ and $\chi$ but not on $a, \tau \in[0,1]$, or $\theta_{0} \in(0,1)$, such that for all $a>a_{0}\left(\theta_{0}\right)$ we have

$$
c_{a} \leqslant K<\infty
$$


Proof. Let us rewrite the equation (23) for $u_{a}$ as

$$
\begin{gathered}
-c_{a} u_{a}^{\prime}-u_{a}^{\prime \prime}+\tau \chi g\left(u_{a}\right) v_{a}^{\prime} u_{a}^{\prime}+\tau \chi g^{\prime}\left(u_{a}\right) u_{a} v_{a}^{\prime} u_{a}^{\prime}=\tau g\left(u_{a}\right) u_{a}\left(1-u_{a}\right)-\frac{\tau \chi}{d} g\left(u_{a}\right) u_{a}\left(v_{a}-u_{a}\right) \\
=\tau g\left(u_{a}\right) u_{a}\left(1-u_{a}+\frac{\chi}{d} u_{a}-\frac{\chi}{d} v_{a}\right) \leqslant \tau g\left(u_{a}\right) u_{a}\left(1-u_{a}+\frac{\chi}{d} u_{a}\right)
\end{gathered}
$$

The last inequality holds if $v_{a} \geqslant 0$. As $g(u)=0$ for $u \leqslant 0$, it follows that $u_{a}$ cannot attain an interior negative minimum on $(-a, a)$ and thus $u_{a} \geqslant 0$, which, in turn, implies that $v_{a} \geqslant 0$ and (26) indeed holds. It also follows from (26) that $u_{a}$ cannot attain an interior maximum at a point where $u_{a} \geqslant(1-\chi / d)^{-1}$. Therefore, $0 \leqslant u_{a} \leqslant(1-\chi / d)^{-1}$ and hence the same bound holds for $v_{a}$. The bound for $\left|v_{a}^{\prime}(x)\right|$ in 24 is then a consequence of 21 .

Next, we show that the speed $c_{a}$ is uniformly bounded from above by using a supersolution argument. The function $u_{a}(x)$ satisfies the inequality

$$
-c_{a} u_{a}^{\prime}-u_{a}^{\prime \prime}+\tau \chi\left[g\left(u_{a}\right)+g^{\prime}\left(u_{a}\right) u_{a}\right] v_{a}^{\prime} u_{a}^{\prime} \leqslant \tau u_{a},
$$

which follows from 26 and the condition $\chi / d<1$. Let us set $\psi_{M}(x)=M e^{-x}$. Then the function $\psi_{M}$ satisfies

$$
\begin{aligned}
-c_{a} \psi_{M}^{\prime}-\psi_{M}^{\prime \prime}+\tau \chi\left[g\left(u_{a}\right)+g^{\prime}\left(u_{a}\right) u_{a}\right] v_{a}^{\prime} \psi_{M}^{\prime} & =\left(c_{a}-1-\tau \chi g\left(u_{a}\right) v_{a}^{\prime}-\tau \chi g^{\prime}\left(u_{a}\right) u_{a} v_{a}^{\prime}\right) \psi_{M} \\
& \geqslant\left(c_{a}-1-K_{0}\right) \psi_{M},
\end{aligned}
$$

with the constant $K_{0}=K_{0}(\chi, d)$, which is uniformly bounded in $a, \tau$ and $\theta_{0}$, chosen so that (using (21)

$$
\chi\left[g\left(u_{a}\right)+g^{\prime}\left(u_{a}\right) u_{a}\right]\left|v_{a}^{\prime}\right| \leqslant \frac{\chi}{\sqrt{d}}\left\|g(\sigma)+g^{\prime}(\sigma) \sigma\right\|_{\infty}\left\|u_{a}\right\|_{\infty} \leqslant K_{0} .
$$

This is possible because of the uniform bounds in (24) and since for $u \notin\left(\theta_{0}, 2 \theta_{0}\right)$ we have $g^{\prime}(u)=0$, while for $u \in\left(\theta_{0}, 2 \theta_{0}\right)$ the following estimate holds:

$$
\left|g^{\prime}(u) u\right|=\frac{u}{\theta_{0}} g_{0}^{\prime}\left(\frac{u-\theta_{0}}{\theta_{0}}\right) \leqslant 2 \max _{1 \leqslant u \leqslant 2}\left|g_{0}^{\prime}(u)\right| .
$$

Now, suppose by contradiction that

$$
c_{a}>2+K_{0}
$$

Then $\psi_{M}$ satisfies

$$
-c_{a} \psi_{M}^{\prime}-\psi_{M}^{\prime \prime}+\tau \chi\left[g\left(u_{a}\right)+g^{\prime}\left(u_{a}\right) u_{a}\right] v_{a}^{\prime} \psi_{M}^{\prime} \geqslant \psi_{M} \geqslant \tau \psi_{M} .
$$

Note that the upper bound on $u_{a}(x)$ in (24) implies that $\psi_{M}(x)>u_{a}(x)$ for $M \geqslant e^{a} /(1-\chi / d)$. Let us define

$$
M_{0}=\inf \left\{M: \psi_{M}(x)>u_{a}(x) \text { for all } x \in[-a, a]\right\} .
$$

Then $M_{0}>0$ and, in addition, $\psi_{M_{0}}(x) \geqslant u_{a}(x)$ for all $x \in[-a, a]$ and there exists $x_{0} \in[-a, a]$ such that $\psi_{M_{0}}\left(x_{0}\right)=u_{a}\left(x_{0}\right)$. However, the difference $\psi_{M_{0}}(x)-u_{a}(x)$ may not attain an interior minimum at $x_{0}$ and $\psi_{M_{0}}(a)>0=u_{a}(a)$. Therefore, $x_{0}=-a$ and thus $M_{0}=e^{-a}$. As a consequence, $\theta_{0}=u_{a}(0) \leqslant \psi_{M_{0}}(0)=e^{-a}$, which is a contradiction if $a$ is sufficiently large. We conclude that (28) is impossible and thus (25) holds with $K=2+K_{0}$. 
A lower bound for the traveling speed

Now, we need a lower bound for $c_{a}$ and an upper bound for $\left\|u_{a}^{\prime}\right\|_{2}$.

LEMMA 2.3 With the assumptions of Lemma 2.2 and (9), there exists a constant $a_{0}\left(\theta_{0}\right)>0$ and $K>0$ which depends only on $d$ and $\chi$ but not on $a>a_{0}, \theta_{0} \in(0,1)$ and $\tau \in[0,1]$, such that for all $a>a_{0}$ and $\theta_{0}<1 / 3$ we have

$$
\begin{aligned}
& c_{a} \geqslant \frac{\sqrt{\tau}}{K}-\frac{K \theta_{0}}{a}, \\
& \tau \int_{-a}^{a} g\left(u_{a}\right) u_{a}\left(1-u_{a}\right)^{2} \mathrm{~d} x+\int_{-a}^{a}\left|u_{a}^{\prime}(x)\right|^{2} \mathrm{~d} x+\int_{-a}^{a}\left|v_{a}^{\prime}(x)\right|^{2} \mathrm{~d} x \leqslant K .
\end{aligned}
$$

Proof. Start with

$$
-c_{a} u_{a}^{\prime}-u_{a}^{\prime \prime}+\tau \chi\left(g\left(u_{a}\right) u_{a} v_{a}^{\prime}\right)^{\prime}=\tau g\left(u_{a}\right) u_{a}\left(1-u_{a}\right),
$$

and integrate on $[-a, a]$ :

$$
c_{a}-u_{a}^{\prime}(a)+u_{a}^{\prime}(-a)-\tau \chi v_{a}^{\prime}(-a)=\tau \int g\left(u_{a}\right) u_{a}\left(1-u_{a}\right) .
$$

Now, multiply (31) by $u_{a}$ and integrate:

$$
\frac{c_{a}}{2}+u_{a}^{\prime}(-a)+\int_{-a}^{a}\left|u_{a}^{\prime}\right|^{2}-\tau \chi v_{a}^{\prime}(-a)-\tau \chi \int_{-a}^{a} g\left(u_{a}\right) u_{a} u_{a}^{\prime} v_{a}^{\prime}=\tau \int_{-a}^{a} g\left(u_{a}\right) u_{a}^{2}\left(1-u_{a}\right) .
$$

Combining the last two equalities, we get

$$
\frac{c_{a}}{2}-u_{a}^{\prime}(a)-\int_{-a}^{a}\left|u_{a}^{\prime}\right|^{2}+\tau \chi \int g\left(u_{a}\right) u_{a} u_{a}^{\prime} v_{a}^{\prime}=\tau \int_{-a}^{a} g\left(u_{a}\right)\left(u_{a}-u_{a}^{2}\right)\left(1-u_{a}\right) .
$$

This can be written as

$$
\tau \int_{-a}^{a} g\left(u_{a}\right) u_{a}\left(1-u_{a}\right)^{2}+\int_{-a}^{a}\left|u_{a}^{\prime}\right|^{2}+u_{a}^{\prime}(a)=\frac{c_{a}}{2}+\tau \chi \int_{-a}^{a} g\left(u_{a}\right) u_{a} u_{a}^{\prime} v_{a}^{\prime} .
$$

However, on the interval $(0, a)$ we have $g\left(u_{a}\right)=0$, and we can find $u_{a}$ explicitly:

$$
u_{a}(x)=\theta_{0} \frac{e^{-c_{a} x}-e^{-c_{a} a}}{1-e^{-c_{a} a}}
$$

so that

Note that for $c_{a}>0$ we have

$$
u_{a}^{\prime}(a)=-\frac{c_{a} \theta_{0} e^{-c_{a} a}}{1-e^{-c_{a} a}}
$$

$$
0 \leqslant \frac{c_{a}}{e^{c_{a} a}-1} \leqslant \frac{1}{a}
$$

while for $c_{a}<0$ we have

$$
0 \leqslant \frac{c_{a}}{e^{c_{a} a}-1}=\frac{a\left|c_{a}\right|}{a\left(1-e^{-\left|c_{a}\right| a}\right)} \leqslant \frac{1+\left|c_{a}\right| a}{a}=\frac{1}{a}+\left|c_{a}\right| .
$$


Therefore, for all $c_{a} \in \mathbb{R}$ we have

$$
\left|u_{a}^{\prime}(a)\right| \leqslant \frac{\theta_{0}}{a}+\left|c_{a}\right| \theta_{0} .
$$

We note that the special case $c_{a}=0$ that we did not treat above can be easily considered separately.

We may use the representation formula $\sqrt{19}$ for $v$ to obtain

$$
v^{\prime}=\tau K_{d} * \bar{u}^{\prime}, \quad\left\|v^{\prime}\right\|_{L^{2}} \leqslant \tau\left\|\bar{u}^{\prime}\right\|_{L^{2}} \leqslant \tau\left\|u^{\prime}\right\|_{L^{2}} .
$$

Using this in 33 we obtain

$$
\tau \int_{-a}^{a} g\left(u_{a}\right) u_{a}\left(1-u_{a}\right)^{2}+\int_{-a}^{a}\left|u_{a}^{\prime}\right|^{2} \leqslant \frac{c_{a}}{2}-u_{a}^{\prime}(a)+\frac{\chi \tau}{1-\chi / d} \int_{-a}^{a}\left|u_{a}^{\prime}\right|^{2} .
$$

It follows that for $0 \leqslant \tau \leqslant 1$ we have, thanks to 35 ,

$$
\tau \int_{-a}^{a} g\left(u_{a}\right) u_{a}\left(1-u_{a}\right)^{2}+M \int_{-a}^{a}\left|u_{a}^{\prime}\right|^{2} \leqslant \frac{c_{a}}{2}+\left|u_{a}^{\prime}(a)\right| \leqslant \frac{c_{a}}{2}+\frac{\theta_{0}}{a}+\theta_{0}\left|c_{a}\right|,
$$

with, according to condition 9 ,

$$
M=1-\frac{\chi}{1-\chi / d}>0 .
$$

In addition, as $u_{a}(-a)=1$ and $u_{a}(0)=\theta_{0}$, there exists a constant $K>0$ which does not depend on $\theta_{0} \in(0,1 / 3)$ such that

$$
\left(\int g\left(u_{a}\right) u_{a}\left(1-u_{a}\right)^{2}\right)\left(\int\left|u_{a}^{\prime}\right|^{2}\right) \geqslant K .
$$

Therefore, provided that $a>a_{0}$ and $\theta_{0} \in(0,1 / 3)$, we have a lower bound for $c_{a}$ :

$$
c_{a} \geqslant c_{0} \sqrt{\tau}-\frac{C \theta_{0}}{a}
$$

with the constants $c_{0}>0$ and $C>0$ which do not depend on the cut-off $\theta_{0}$. This is the bound in 299, while the bounds in (30) follow from the upper bound (25) for the speed, 36) and 38.

\section{The homotopy argument}

We may now finish the proof of Proposition 2.1 using a homotopy argument. The a priori bounds obtained in Lemmas 2.2 and 2.3 allow us to use the Leray-Schauder topological degree argument to prove existence of solutions to the problem (16), (17), (19) with the normalization (22) on the bounded interval $D_{a}=(-a, a)$. This method of construction of traveling wave solutions goes back to [3]. We introduce a map (we suppress the subscript $a$ now, resurrecting the subscript $\tau$ for the homotopy parameter)

$$
\mathcal{K}_{\tau}:(c, u, v) \rightarrow\left(\theta_{\tau}, U_{\tau}, V_{\tau}\right)
$$

as the solution operator of the linear system

$$
\left\{\begin{array}{l}
-c U_{\tau}^{\prime}-U_{\tau}^{\prime \prime}+\tau \chi\left(g(u) U_{\tau} v^{\prime}\right)^{\prime}=\tau g(u) u(1-u), \\
-d V_{\tau}^{\prime \prime}+V_{\tau}=\tau \bar{u} .
\end{array}\right.
$$


The boundary conditions for $U_{\tau}$ are as in (17):

$$
U_{\tau}(-a)=1, \quad U_{\tau}(a)=0,
$$

while $V_{\tau}$ is given explicitly as before by

$$
V_{\tau}(x)=\tau \int_{-\infty}^{\infty} K_{d}(|x-\xi|) \bar{u}(\xi) \mathrm{d} \xi, \quad K_{d}(\xi)=\frac{e^{-|\xi| / \sqrt{d}}}{2 \sqrt{d}}
$$

where $\bar{u}(x)$ is again the extension of $u(x)$ to the whole real line as in (18).

The number $\theta_{\tau}$ is defined by

$$
\theta_{\tau}=\theta_{0}-\max _{x \geqslant 0} u(x)+c .
$$

The operator $\mathcal{K}_{\tau}$ is a mapping of the Banach space $X=\mathbb{R} \times C^{1, \alpha}\left(D_{a}\right) \times C^{2, \alpha}\left(D_{a}\right)$, equipped with the norm $\|(c, u, v)\|_{X}=\max \left(|c|,\|u\|_{C^{1, \alpha}\left(D_{a}\right)},\|v\|_{C^{1, \alpha}\left(D_{a}\right)}\right)$, onto itself. A solution $s_{\tau}=$ $\left(c_{\tau}, u_{\tau}, v_{\tau}\right)$ of the finite interval problem (16), (17), (19), (22) is a fixed point of $\mathcal{K}_{\tau}$ and satisfies $\mathcal{K}_{\tau} s_{\tau}=s_{\tau}$, and vice versa: a fixed point of $\mathcal{K}_{\tau}$ provides a solution. Hence, in order to establish the existence of a solution to (16), (17), (19) together with the normalization (22), it suffices to show that the kernel of the operator $\mathcal{F}_{\tau}=\operatorname{Id}-\mathcal{K}_{\tau}$ is not trivial. The standard elliptic regularity theory implies that the operator $\mathcal{K}_{\tau}$ is compact and depends continuously on the parameter $\tau \in[0,1]$. Thus we may apply the Leray-Schauder topological degree theory. Let us introduce a ball $B_{M}=\left\{\|(c, u, v)\|_{X} \leqslant M\right\}$. Then Lemmas 2.2 and 2.3 show that the operator $\mathcal{F}_{\tau}$ does not vanish on the boundary $\partial B_{M}$ with $M$ sufficiently large for any $\tau \in[0,1]$. It remains to show that the degree $\operatorname{deg}\left(\mathcal{F}_{1}, B_{M}, 0\right)$ in $\bar{B}_{M}$ is not zero. However, the homotopy invariance property of the degree implies that $\operatorname{deg}\left(\mathcal{F}_{\tau}, B_{M}, 0\right)=\operatorname{deg}\left(\mathcal{F}_{0}, B_{M}, 0\right)$ for all $\tau \in[0,1]$. Moreover, the degree at $\tau=0$ can be computed explicitly as the operator $\mathcal{F}_{0}$ is given by

$$
\mathcal{F}_{0}(c, u, v)=\left(\max _{x \geqslant 0} u(x)-\theta_{0}, u-u_{0}^{c}, v\right) .
$$

Here the function $u_{0}^{c}(x)$ solves

$$
\frac{\mathrm{d}^{2} u_{0}^{c}}{\mathrm{~d} x^{2}}+c \frac{\mathrm{d} u_{0}^{c}}{\mathrm{~d} x}=0, \quad u_{0}^{c}(-a)=1, u_{0}^{c}(a)=0,
$$

and is given by

The mapping $\mathcal{F}_{0}$ is homotopic to

$$
u_{0}^{c}(x)=\frac{e^{-c x}-e^{-c a}}{e^{c a}-e^{-c a}}
$$

$$
\Phi(c, u, v)=\left(\max _{x \geqslant 0} u_{0}^{c}(x)-\theta_{0}, u-u_{0}^{c}, v\right),
$$

which in turn is homotopic to

$$
\tilde{\Phi}(c, u, v)=\left(u_{0}^{c}(0)-\theta_{0}, u-u_{0}^{c_{*}^{0}}, v\right),
$$

where $c_{*}^{0}$ is the unique number so that $u_{0}^{c_{*}}(0)=\theta_{0}$. The degree of the mapping $\tilde{\Phi}$ is the product of the degrees of each component. The last two have degree 1 , and the first -1 , as the function $u_{0}^{c}(0)$ is decreasing in $c$. Thus $\operatorname{deg} \mathcal{F}_{0}=-1$ and hence $\operatorname{deg} \mathcal{F}_{1}=-1$ so that the kernel of Id $-\mathcal{K}_{1}$ is not empty. This finishes the proof of Proposition 2.1 


\section{Identification of the limit as $a \rightarrow+\infty$}

In this section we first let $a \rightarrow+\infty$ constructing traveling waves with a positive cut-off $\theta_{0}>0$. In the second step we remove the cut-off and obtain traveling waves for the Fisher-KPP birth rate. At this stage we only prove a loose lower bound on $c_{*}$; the more precise bound stated in Theorem 1.1 is proved in Section 4

\section{Passage to the whole line with a cut-off}

We now prove Proposition 1.2 Having established the existence of a solution $\left(c_{a}, u_{a}, v_{a}\right)$ of 16 , [17), (19), 222) on a finite interval we now let $a \rightarrow+\infty$ and show that $\left(c_{a}, u_{a}, v_{a}\right)$ converges to a traveling wave $(c, u, v)$. The $L^{2}$-bound for $u^{\prime}(x)$ and $v^{\prime}(x)$ in Lemma 2.3 together with the uniform bounds in Lemma 2.2 and the elliptic regularity imply that there exists a sequence $a_{n} \rightarrow+\infty$ so that $c_{n}=c_{a_{n}}$ converges to a limit $c_{*}\left(\theta_{0}\right)$ and the functions $u_{n}=u_{a_{n}}$ and $v_{n}=v_{a_{n}}$ converge locally uniformly together with their derivatives to the limits $u\left(x ; \theta_{0}\right)$ and $v\left(x ; \theta_{0}\right)$. The functions $u(x)$ and $v(x)$ satisfy (we drop the dependence on $\theta_{0}$ in the notation)

$$
\left\{\begin{array}{l}
-c_{*} u^{\prime}-u^{\prime \prime}+\chi\left(g(u) u v^{\prime}\right)^{\prime}=g(u) u(1-u), \\
-d v^{\prime \prime}+v=u
\end{array}\right.
$$

and

$$
v(x)=\int_{-\infty}^{\infty} K_{d}(|x-\xi|) u(\xi) \mathrm{d} \xi, \quad K_{d}(\xi)=\frac{e^{-|\xi| / \sqrt{d}}}{2 \sqrt{d}} .
$$

Furthermore, the lower bound of Lemma 2.3 yields $c_{*}\left(\theta_{0}\right) \geqslant 1 / K$, where $K$ is a positive constant that only depends on $d$ and $\chi$. In particular, $c_{*}$ is positive.

It remains to prove that $u(x)$ and $v(x)$ satisfy the boundary conditions (2) and, because of (43), it is sufficient to verify them for the function $u(x)$ only. The $L^{2}$-bound for the gradient of $u$ in Lemma 2.3 and elliptic regularity imply that the function $u(x)$ has limits as $x \rightarrow \pm \infty$ :

$$
u_{l}=\lim _{x \rightarrow-\infty} u(x), \quad u_{r}=\lim _{x \rightarrow+\infty} u(x) .
$$

The functions $u_{a}(x)$ are given by an explicit expression (34) on the interval $0 \leqslant x \leqslant a$. Therefore, the limit $u(x)$ is given by

$$
u(x)=\theta_{0} e^{-c_{*} x} \quad \text { for all } x \geqslant 0 .
$$

As $c_{*}>0$, it follows that $u_{r}=0$.

Next, we show that $u_{l}=1$ when $\theta_{0}$ is sufficiently small. We first note that according to the maximum principle the function $u_{a}$ cannot attain a minimum at a point $x$ where $u_{a}(x) \leqslant \theta_{0}$. Therefore, $u_{a} \geqslant \theta_{0}$ for $x \in(-a, 0)$ and thus $u_{l} \geqslant \theta_{0}$. On the other hand, the uniform bound

$$
\int_{-a}^{a} g\left(u_{a}\right) u_{a}\left(1-u_{a}\right)^{2} \mathrm{~d} x \leqslant K
$$

in Lemma 2.3 implies that the limit $u(x)$ satisfies

$$
\int_{-\infty}^{\infty} g(u) u(1-u)^{2} \mathrm{~d} x \leqslant K .
$$


Therefore, either $u_{l}=1$ or $u_{l} \in\left[0, \theta_{0}\right]$. The previous argument implies that the only two possibilities are $u_{l}=\theta_{0}$ and $u_{l}=1$. Let us assume that $u_{l}=\theta_{0}$ and find a contradiction when $\theta_{0}$ is sufficiently small. With this assumption we integrate the first equation in (42) once to get

$$
c_{*} \theta_{0}=\int_{-\infty}^{\infty} g(u) u(1-u) \mathrm{d} x
$$

Multiplying the same equation by $u$ and integrating leads to

$$
\begin{aligned}
\frac{c_{*} \theta_{0}^{2}}{2}+\int_{-\infty}^{\infty}\left|u^{\prime}\right|^{2} \mathrm{~d} x-\chi \int_{-\infty}^{\infty} g(u) u u^{\prime} v^{\prime} \mathrm{d} x & =\int_{-\infty}^{\infty} g(u) u^{2}(1-u) \mathrm{d} x \\
& =c_{*} \theta_{0}-\int_{-\infty}^{\infty} g(u) u(1-u)^{2} \mathrm{~d} x
\end{aligned}
$$

Using the $L^{\infty}$-bound for $u$ and since $\left\|v^{\prime}\right\|_{2} \leqslant\left\|u^{\prime}\right\|_{2}$ we get, still using condition (9),

$$
\frac{c_{*} \theta_{0}^{2}}{2}+K \int_{-\infty}^{\infty}\left|u^{\prime}\right|^{2} \mathrm{~d} x+\int_{-\infty}^{\infty} g(u) u(1-u)^{2} \mathrm{~d} x \leqslant c_{*} \theta_{0},
$$

with $K>0$, as in the computation leading to 38 . Note that since $u_{l}=u(0)=\theta_{0}$ and $u(x)$ cannot attain a local minimum at a value below $\theta_{0}$, the function $u(x)$ attains its maximum at some point $x_{M}$-otherwise, $g(u) \equiv 0$ and $c_{*}=0$, which would be a contradiction. For the same reason, $u_{M}=u\left(x_{M}\right)>\theta_{0}$ since the integral on the right side of 46 is positive because $c_{*}>0$. Observe that if $u_{M}>1 / 2$ and $u_{l}=\theta_{0}<1 / 3$, then there exists $K_{1}>0$ which does not depend on $\theta_{0}$ so that

$$
\int_{-\infty}^{\infty}\left|u^{\prime}\right|^{2}+\int_{-\infty}^{\infty} g(u) u(1-u)^{2} \geqslant K_{1}
$$

Therefore, as $c_{*}$ is bounded from above, it follows from (48) that there exists $\alpha_{0}>0$ so that if $\theta_{0} \in\left(0, \alpha_{0}\right)$ then $\theta_{0}<u_{M}<1 / 2$.

Next, assume that $\theta_{0} \in\left(0, \alpha_{0}\right)$ and integrate the first equation in 42 between $-\infty$ and $x_{M}$ to get

$$
-c_{*}\left(u_{M}-\theta_{0}\right)+\chi g\left(u_{M}\right) u_{M} v^{\prime}\left(u_{M}\right)=\int_{-\infty}^{x_{M}} g(u) u(1-u) \mathrm{d} x .
$$

As $u_{M}<1 / 2$, the right side above is positive. In addition, we have $\left\|v^{\prime}\right\|_{L^{\infty}} \leqslant C\|u\|_{\infty}=C u_{M}$ and

$$
g(u)=g_{0}\left(\frac{u-\theta_{0}}{\theta_{0}}\right) \leqslant \frac{C\left(u-\theta_{0}\right)}{\theta_{0}}
$$

for $u \geqslant \theta_{0}$. Then (49) implies

$$
-c_{*}\left(u_{M}-\theta_{0}\right)+\frac{C \chi\left(u_{M}-\theta_{0}\right) u_{M}^{2}}{\theta_{0}} \geqslant 0 .
$$

Therefore, as $c_{*}>0$ and $u_{M}>\theta_{0}$, we have

$$
u_{M}^{2} \geqslant K \theta_{0} \quad \text { with } K>0 .
$$


In particular, $u_{M} \geqslant 2 \theta_{0}$ when $\theta_{0}$ is sufficiently small. Let $x_{0}$ be the first point to the left of $x_{M}$ such that $u\left(x_{0}\right)=u_{M} / 2$, that is, $u(x) \in\left[u_{M} / 2, u_{M}\right]$ for all $x \in\left(x_{0}, x_{M}\right)$ and $g(u(x))=1$ on this interval. Set $L=x_{M}-x_{0}$. Then we have, using [48],

$$
c_{*} \theta_{0} \geqslant K \int_{x_{0}}^{x_{M}}\left|u^{\prime}\right|^{2}+\int_{x_{0}}^{x_{M}} g(u) u(1-u)^{2} \geqslant C\left[\frac{u_{M}^{2}}{L}+u_{M} L\right] \geqslant C u_{M}^{3 / 2} .
$$

It follows that $u_{M} \leqslant C \theta_{0}^{2 / 3}$, which contradicts 50 . This contradiction shows that $u_{l}=\theta_{0}$ is impossible when $\theta_{0}$ is sufficiently small. Therefore, $u_{l}=1$. This finishes the proof of Proposition 1.2

Proof of Proposition 1.3 We now indicate the additional arguments necessary to arrive at the statement of Proposition 1.3, that is, how existence of traveling waves can be deduced under the weaker restriction (4) on the chemotaxis parameter $\chi$.

The entire proof above of Proposition 1.2 goes through with the general assumption [12] on $g$. We now indicate how we can take advantage of the property

$$
g+\sigma g^{\prime} \leqslant 1+\alpha .
$$

First, the upper bound on $c_{*}$ in (7) follows clearly from the value $K_{0}$ computed in 27).

Now, we prove gradient and "reaction" bounds in (6). To do that we use equation (33), and the key point is to handle the right hand side more carefully with the help of (51): we split the integral as

$$
\chi \int_{-a}^{a} \tau g\left(u_{a}\right) u_{a} u_{a}^{\prime} v_{a}^{\prime}=\chi \tau \int_{-a}^{a}\left[g\left(u_{a}\right) u_{a}-1\right] u_{a}^{\prime} v_{a}^{\prime}+\chi \tau \int_{-a}^{a} u_{a}^{\prime} v_{a}^{\prime}
$$

We treat separately the two terms on the right side.

Using the equation on $v$ in 111, which now also has the small density cut-off, we have

$$
\begin{aligned}
\left(\chi \tau \int_{-a}^{a} u_{a}^{\prime} v_{a}^{\prime}\right)^{2} & \leqslant \chi^{2} \int_{-a}^{a}\left(u_{a}^{\prime}\right)^{2} \int_{-a}^{a}\left(v_{a}^{\prime}\right)^{2} \leqslant \chi^{2} \int_{-a}^{a}\left(u_{a}^{\prime}\right)^{2} \int_{-a}^{a}\left[\left(\tau g\left(u_{a}\right)+u_{a} \tau g^{\prime}\left(u_{a}\right)\right) u_{a}^{\prime}\right]^{2} \\
& \leqslant \chi^{2}(1+\alpha)^{2}\left(\int_{-a}^{a}\left(u_{a}^{\prime}\right)^{2}\right)^{2} .
\end{aligned}
$$

This term is nicely absorbed for $\chi<1$ and $\alpha$ (or, equivalently, $\theta_{0}$ ) small enough by the corresponding term on the left hand side of 33 .

For the other term, we introduce the function

$$
h(u)=\int_{1}^{u}[g(\sigma) \sigma-1] \mathrm{d} \sigma \quad \text { for } 0 \leqslant u \leqslant 1
$$

and with $h(u)=0$ for $u \geqslant 1$. Note that

$$
0 \leqslant h(u) \leqslant \frac{1}{2}(1-u)^{2}, \quad h(1)=0 .
$$


We write

$$
\begin{aligned}
\chi \tau \int_{-a}^{a}\left[g\left(u_{a}\right) u_{a}-1\right] u_{a}^{\prime} v_{a}^{\prime} & =\chi \tau \int_{-a}^{a} h\left(u_{a}\right)^{\prime} v_{a}^{\prime} \\
& =\chi \tau \int_{-a}^{a} h\left(u_{a}\right)\left(-v_{a}\right)^{\prime \prime}+\left.\chi \tau h\left(u_{a}\right) v_{a}^{\prime}\right|_{x=a}-\left.\chi \tau h\left(u_{a}\right) v_{a}^{\prime}\right|_{x=-a} \\
& \leqslant \frac{\chi \tau}{d} \int_{-a}^{a} h\left(u_{a}\right)\left(g\left(u_{a}\right) u_{a}-v_{a}\right) \leqslant \tau \frac{\chi}{2 d} \int_{-a}^{a}\left(1-u_{a}\right)^{2} g\left(u_{a}\right) u_{a},
\end{aligned}
$$

because $v_{a}^{\prime}(a)=\left(K_{d}^{\prime} * \bar{u}_{a}\right)(a) \leqslant 0$ for $a$ sufficiently large. Consequently,

$$
\begin{aligned}
\tau \int_{-a}^{a} g\left(u_{a}\right) u_{a}\left(1-u_{a}\right)^{2}+ & \int_{-a}^{a}\left|u_{a}^{\prime}\right|^{2}+u_{a}^{\prime}(a)=\frac{c_{a}}{2}+\tau \chi \int_{-a}^{a} g\left(u_{a}\right) u_{a} u_{a}^{\prime} v_{a}^{\prime} \\
& \leqslant \frac{c_{a}}{2}+\chi(1+\alpha) \int_{-a}^{a}\left(u_{a}^{\prime}\right)^{2}+\tau \frac{\chi}{2 d} \int_{-a}^{a}\left(1-u_{a}\right)^{2} g\left(u_{a}\right) u_{a} .
\end{aligned}
$$

It follows that

$$
\tau\left(1-\frac{\chi}{2 d}\right) \int_{-a}^{a} g\left(u_{a}\right) u_{a}\left(1-u_{a}\right)^{2}+(1-\chi(1+\alpha)) \int_{-a}^{a}\left|u_{a}^{\prime}\right|^{2}+u_{a}^{\prime}(a) \leqslant \frac{c_{a}}{2},
$$

and $u_{a}^{\prime}(a)$ is still bounded by 35 .

Thus if $\chi<\min (1, d)$ (recall the upper control by $d$ is needed for the $L^{\infty}$ bound) and $\theta_{0}$ is small enough such that $\chi(1+\alpha)<1$, the quantities of the left hand side are controlled by that of the right hand side and we can go on with the proof and conclude as before.

\section{Removal of the cut-off}

Here we remove the cut-off, letting the parameter $\theta_{0}$ vanish, and prove Theorem 1.1 . The traveling waves $\left(c\left(\theta_{0}\right), u\left(x ; \theta_{0}\right), v\left(x ; \theta_{0}\right)\right)$, constructed in Proposition 1.3 for $\theta_{0}>0$, are translationally invariant and have the left and right limits $u_{l}=v_{l}=1, u_{r}=v_{r}=0$. Therefore, we may translate them and fix the shift so that $u\left(0 ; \theta_{0}\right)=1 / 2$. The uniform estimates in the same proposition allow us to let $\theta_{0, n} \rightarrow 0$ along a subsequence, so that the traveling wave speeds $c_{n}=c_{*}\left(\theta_{0, n}\right)$ converge to a limit $c_{*}>0$, and the functions $u\left(x ; \theta_{0, n}\right)$ and $v\left(x ; \theta_{0, n}\right)$ converge to $u(x)$ and $v(x)$. We also have $g\left(u_{n}\right) \rightarrow \Psi(x)$ with $\Psi(x) \equiv 1$ on the set $\{u(x) \neq 0\}$. In addition, the limits satisfy the system $(3)$ :

$$
\begin{aligned}
& -c_{*} u^{\prime}-u^{\prime \prime}+\chi\left(\Psi(x) u v^{\prime}\right)^{\prime}=\Psi(x) u(1-u), \\
& -d v^{\prime \prime}+v=\Psi(x) u,
\end{aligned}
$$

and the functions $u$ and $v$ are still related by (43). Moreover, as the function $p(u)=g(u) u$ is globally Lipschitz, the functions $u\left(x ; \theta_{0, n}\right)$ and $v\left(x ; \theta_{0, n}\right)$ are uniformly bounded in $C^{2, \alpha}(\mathbb{R})$ and thus so are the limits $u$ and $v$. Therefore, we have $u>0$ and thus $\Psi(x) \equiv 1$ and $u$ and $v$ actually satisfy the system (3):

$$
\begin{aligned}
& -c_{*} u^{\prime}-u^{\prime \prime}+\chi\left(u v^{\prime}\right)^{\prime}=u(1-u), \\
& -d v^{\prime \prime}+v=u .
\end{aligned}
$$


It remains to verify that $u$ and $v$ satisfy the boundary conditions (2) at infinity. As in the case with $\theta_{0}>0$ it suffices to ensure that $u(x)$ has the left and right limits $u_{l}=1$ and $u_{r}=0$, respectively. Once again, existence of the limits at infinity follows from the $L^{2}$-bound on the gradient

$$
\int_{-\infty}^{\infty}\left|u^{\prime}(x)\right|^{2} \mathrm{~d} x \leqslant K
$$

and standard elliptic regularity estimates. Moreover, in the limit $\theta_{0} \rightarrow 0$ the estimate 45 becomes

$$
\int_{-\infty}^{\infty} u(1-u)^{2} \mathrm{~d} x \leqslant K<\infty
$$

As a consequence, the only possible values for $u_{l}$ and $u_{r}$ are 0 and 1 , hence in order to show that $u_{l}=1$ and $u_{r}=0$ it suffices to show that $u_{l}>u_{r}$. Integrating the first equation in 55 we obtain

$$
c_{*}\left(u_{l}-u_{r}\right)=\int u(1-u),
$$

while multiplying the same equation by $u$ and integrating leads to

$$
\frac{c_{*}\left(u_{l}^{2}-u_{r}^{2}\right)}{2}+\int\left|u^{\prime}\right|^{2}-\chi \int u u^{\prime} v^{\prime}=\int u^{2}(1-u)=c_{*}\left(u_{l}-u_{r}\right)-\int u(1-u)^{2} .
$$

As before, we conclude that

$$
\frac{c_{*}\left(u_{l}^{2}-u_{r}^{2}\right)}{2}+\int u(1-u)^{2}+M \int\left|u^{\prime}\right|^{2} \leqslant c_{*}\left(u_{l}-u_{r}\right),
$$

which may be rewritten as

$$
\int u(1-u)^{2}+M \int\left|u^{\prime}\right|^{2} \leqslant c_{*}\left(u_{l}-u_{r}\right)\left(1-\frac{u_{l}+u_{r}}{2}\right) .
$$

As $u(0)=1 / 2$ the left side is strictly positive. Moreover, $c_{*}>0$ and $\left(u_{l}+u_{r}\right) / 2 \leqslant 1$. As a consequence, $u_{l}>u_{r}$, thus $u_{l}=1, u_{r}=0$, and the proof of the existence part of Theorem 1.1 is complete.

\section{A lower bound for the traveling speed}

We now obtain a more precise lower bound for the propagation speed $c_{*}$ in Theorem 1.1 . To do so, we consider a more general birth term $f(u)$ in place of $u(1-u)$ in equation (3). We do not expect more difficulties in the proof of the existence part of Theorem 1.1 as long as $f(u)$ is of the KPP type:

$$
f(0)=f(1)=0, f(u)>0 \text { for } 0 \leqslant u \leqslant 1, f(u)<0 \text { for } u \geqslant 1 \text { and } f^{\prime}(0)=\sup _{u \geqslant 0} \frac{f(u)}{u}>0 .
$$

Then we have 
Proposition 4.1 Any traveling wave solution of $\sqrt{2}-\sqrt{3}$ in $\dot{H}^{1}(\mathbb{R})$ with the nonlinearity $f$ satisfying (56) and such that $u, v \geqslant 0$, and

$$
\int u(1-u)^{2} \mathrm{~d} x<\infty
$$

satisfies $c \geqslant 2 \sqrt{f^{\prime}(0)}$.

Proof. Consider a traveling wave $(c, u, v)$ and choose a sequence $x_{n}$ that increases to $\infty$ as $n \rightarrow \infty$. Note that $u\left(x+x_{n}\right) \rightarrow 0$ uniformly in $x \in[R, \infty)$ for all $R \in \mathbb{R}$ as $n \rightarrow \infty$. Indeed, choosing $A>0$ large enough so that $u(x) \leqslant 1 / 2$ for $x \geqslant A$, we deduce from $\sqrt[57]{ }$ that $u \in L^{1}(A, \infty)$ and thus we may write

$$
u^{2}(x)=-2 \int_{x}^{\infty} u^{\prime} \leqslant 2\left(\int_{A}^{\infty} u^{2}\right)^{1 / 2}\left(\int_{A}^{\infty} u^{\prime 2}\right)^{1 / 2} \underset{\theta_{0} \rightarrow 0}{\longrightarrow} 0
$$

so that in particular $u\left(x_{n}\right) \rightarrow 0$ as $n \rightarrow \infty$.

Next, set $u_{n}(x)=u\left(x+x_{n}\right) / u\left(x_{n}\right)$ and $v_{n}(x)=v\left(x+x_{n}\right)$. These functions satisfy

$$
\left\{\begin{array}{l}
-u_{n}^{\prime \prime}-c u_{n}^{\prime}+\chi\left(v_{n}^{\prime} u_{n}\right)^{\prime}=f\left(u\left(x_{n}\right) u_{n}\right) / u\left(x_{n}\right), \\
-d v_{n}^{\prime \prime}+v_{n}=u\left(x+x_{n}\right) .
\end{array}\right.
$$

The right side in the equation on $u_{n}$ in 58 is bounded by $f^{\prime}(0) u_{n}$. Therefore we use elliptic regularity and, up to extracting a subsequence, we know that $u_{n} \rightarrow u_{\infty}$ and $v_{n} \rightarrow v_{\infty}$ as $n \rightarrow \infty$ in $\mathcal{C}_{\text {loc }}^{2}(\mathbb{R})$. These functions satisfy

$$
\left\{\begin{array}{l}
-u_{\infty}^{\prime \prime}-c u_{\infty}^{\prime}+\chi\left(v_{\infty}^{\prime} u_{\infty}\right)^{\prime}=f^{\prime}(0) u_{\infty} \\
-d v_{\infty}^{\prime \prime}+v_{\infty}=0
\end{array}\right.
$$

As $v_{\infty}$ is nonnegative and bounded, we necessarily have $v_{\infty} \equiv 0$.

Furthermore, as $u_{\infty}(0)=1$ and $u_{\infty} \geqslant 0$, the maximum principle yields $u_{\infty}>0$. Thus we can explicitly solve the first equation and the solution can only be of the exponential type: $u_{\infty}(x)=$ $\mu e^{-\lambda x}$. Inserting such a $\lambda$ in the equation for $u_{\infty}$ we find $-\lambda^{2}+c \lambda=f^{\prime}(0)$. Hence we have proved that necessarily $c \geqslant 2 \sqrt{f^{\prime}(0)}$.

\section{Time evolution problem}

We now consider the problem

$$
\left\{\begin{array}{l}
u_{t}-u_{x x}+\chi\left(u v_{x}\right)_{x}=u(1-u), \\
-d v_{x x}+v=u, \\
u(t=0)=u_{0}, \quad \text { with compact support, } 0 \leqslant u_{0}(x) \leqslant(1-\chi / d)^{-1} .
\end{array}\right.
$$

The maximum principle, as already used earlier, implies that we have the uniform bounds

$$
0 \leqslant u(x), v(x) \leqslant \frac{d}{d-\chi}, \quad\left|v_{x}(t, x)\right|,\left|v_{x x}(t, x)\right| \leqslant K .
$$

Our goal in this section is to prove two kinds of results on this problem. First, we assume that $\chi$ satisfies the conditions of existence of traveling waves. Then we derive some bounds expressing that in the long time limit, the solution converges to 1 on compact sets. Secondly, we show that, under the (weaker) linear stability condition on $\chi$, the state 1 is in fact nonlinearly asymptotically stable. 
5.1 The long time limit of $u(t, x)$

We have the following

Theorem 5.1 Assume $\chi \leqslant \min (1, d)$. There exist $C>0$ and $\varepsilon_{0}>0$ such that for any $\varepsilon \in\left(0, \varepsilon_{0}\right)$ there exists a time $t_{0}$ such that for all $T>t_{0}$ the following holds. There exists a set $B \subset[T, 2 T]$ of exceptional times with $|B| \leqslant C / \varepsilon$ such that for all nonexceptional $t \in[T, 2 T] \cap B^{c}$ and all $p \in[0,1)$ we have

$$
\left|\left\{x: u(t, x)|1-u(t, x)|^{2} \geqslant \varepsilon^{p}\right\}\right| \leqslant C \varepsilon^{1-p} \int u(t, x) \mathrm{d} x .
$$

The constant $C>0$ in Theorem 5.1 does not depend on the time $T$. Therefore, the total set $B$ of "bad" times between a (large) time $T$ and $2 T$ is bounded independent of $T$. The right side of (61) may be loosely interpreted as the size of the support of the function $u(t, x)$ (disregarding the fact that $u(t, x)$ has an infinite support). Thus, 61) may be interpreted as saying that for large times the fraction of the support of $u(t, x)$ where $u(t, x)$ is far from 1 is negligible, except for a (relatively) small set of bad times.

We first prove the following proposition.

Proposition 5.2 Assume that (4) holds and let the initial data $u_{0}(x) \not \equiv 0$ be compactly supported, with $0 \leqslant u_{0}(x) \leqslant 1$. There exist two constants $K_{1}$ and $K_{2}$ which do not depend on the initial data, and a time $t_{0}$ such that

$$
K_{1}\left(t-t_{0}\right) \leqslant \int u(t, x) \mathrm{d} x \leqslant K_{2}\left(t_{0}+t\right) .
$$

Proof. First, let $u$ and $v$ be solutions of 60 and consider the function $\psi(t, x)=M e^{-\lambda(x-\xi t)}$. It satisfies the inequality

$$
\begin{aligned}
\psi_{t}-\psi_{x x}+\chi v_{x} \psi_{x}+\chi v_{x x} \psi-\psi(1-\psi) \geqslant \psi_{t}-\psi_{x x}+\chi v_{x} \psi_{x}-\frac{\chi}{d}(u-v) \psi-\psi \\
\geqslant \psi_{t}-\psi_{x x}-K\left|\psi_{x}\right|-K \psi=\left(\lambda \xi \psi-\lambda^{2}-K-K \lambda\right) \psi \geqslant 0 .
\end{aligned}
$$

This last inequality holds provided that $\xi$ is sufficiently large and $\lambda$ is chosen appropriately. Therefore, we may also take $M$ large enough so that $\psi_{M}(t, x)$ is a supersolution for $u(t, x)$. Similarly, $\phi_{M}(t, x)=M e^{\lambda(x+\xi t)}$ is a supersolution for $u$. Therefore,

$$
u(t, x) \leqslant \min \left(M e^{-\lambda(x-\xi t)}, M e^{\lambda(x+\xi t)}\right)
$$

and integrating in $x$ gives

$$
\int_{\mathbb{R}} u(t, x) \mathrm{d} x \leqslant C\left(t+t_{0}\right)
$$

Thus the upper bound of the proposition is proved.

To obtain a lower bound on $\|u(t)\|_{L^{1}}$ we proceed as in the traveling wave case. We have

$$
\frac{\mathrm{d}}{\mathrm{d} t} \int\left(u-u^{2} / 2\right)=\int u_{x}^{2}+\int u(1-u)^{2}-\chi \int u_{x} v_{x} u .
$$


534

G. NADIN ET AL.

The last integral on the right side may be split as

$$
\chi \int u u_{x} v_{x} \mathrm{~d} x=\chi \int(u-1) u_{x} v_{x} \mathrm{~d} x+\chi \int u_{x} v_{x} \mathrm{~d} x
$$

The second term is bounded as

$$
\left(\int u_{x} v_{x} \mathrm{~d} x\right)^{2} \leqslant \int u_{x}^{2} \mathrm{~d} x \int v_{x}^{2} \mathrm{~d} x \leqslant\left(\int u_{x}^{2} \mathrm{~d} x\right)^{2},
$$

while the first one satisfies

$$
\chi \int(u-1) u_{x} v_{x} \mathrm{~d} x=\frac{\chi}{2} \int\left((u-1)^{2}\right)_{x} v_{x} \mathrm{~d} x=\frac{\chi}{2 d} \int(u-1)^{2}(u-v) \mathrm{d} x \leqslant \frac{\chi}{2 d} \int(u-1)^{2} u \mathrm{~d} x
$$

Using the last two inequalities in 63 leads to

$$
\begin{aligned}
\frac{\mathrm{d}}{\mathrm{d} t} \int\left(u-u^{2} / 2\right) & \geqslant \int u_{x}^{2}+\int u(1-u)^{2}-\chi \int u_{x}^{2}-\frac{\chi}{2 d} \int(u-1)^{2} u \mathrm{~d} x \\
& \geqslant M \int u_{x}^{2}+M \int u(1-u)^{2} .
\end{aligned}
$$

Integrating in time and combining this with the upper bound in 62 we obtain

$$
\int_{0}^{T} \int u_{x}^{2}+\int_{0}^{T} \int u(1-u)^{2} \leqslant \frac{1}{M}\left[\int u(T, x) \mathrm{d} x-\int\left(u_{0}-u_{0}^{2} / 2\right)\right] \leqslant C(1+T) .
$$

Note that if at some time $t \in[0, T]$ there exists $x_{0}$ such that $u\left(t, x_{0}\right)>1 / 2$ then

$$
M \int u_{x}^{2}(t, x) \mathrm{d} x+M \int u(t, x)(1-u(t, x))^{2} \mathrm{~d} x \geqslant K .
$$

On the other hand, if $0 \leqslant u(t, x) \leqslant 1 / 2$ for all $x \in \mathbb{R}$ then

$$
\int u(t, x)(1-u(t, x))^{2} \mathrm{~d} x \geqslant \frac{1}{4} \int u(t, x) \mathrm{d} x .
$$

Let $\mathcal{A}_{T}=\{t \in[0, T]: 0 \leqslant u(t, x) \leqslant 1 / 2$ for all $x \in \mathbb{R}\}$. It follows from the above that there exists a constant $K>0$ so that

$$
\int u(T, x) \geqslant \int_{\mathcal{A}_{T}^{c}} K \mathrm{~d} x+K \int_{\mathcal{A}_{T}}\left(\int u(t, x) \mathrm{d} x\right) \mathrm{d} t .
$$

As a consequence, the function

$$
W(t)=\int u(t, x) \mathrm{d} x
$$

satisfies

$$
W(T) \geqslant \int_{\mathcal{A}_{T}^{c}} K \mathrm{~d} x+K \int_{\mathcal{A}_{T}} W(t) \mathrm{d} t \geqslant K \int_{0}^{T} \min (1, W(t)) \mathrm{d} t
$$


for all $T \geqslant 0$. In addition, $W(t)$ is locally Lipschitz in time:

$$
\left|W_{t}(t)\right|=\left|\int u(t, x)(1-u(t, x)) \mathrm{d} x\right| \leqslant M \int u(t, x) \mathrm{d} x \leqslant C(1+t) .
$$

Therefore, in particular, there exists $\tau_{0}$ so that $W(t) \geqslant W(0) / 2>0$ for $0 \leqslant t \leqslant \tau_{0}$ and thus there exists $k_{0}>0$ (which depends on the initial data) such that for $T \geqslant \tau_{0}$,

$$
W(T) \geqslant K \int_{0}^{T} \min (1, W(t)) \mathrm{d} t \geqslant K \int_{0}^{\tau_{0}} \min (1, W(t)) \mathrm{d} t \geqslant k_{0} .
$$

Going back to (66) we see that

$$
W(T) \geqslant K\left|\mathcal{A}_{T}^{c}\right|+k_{0} K\left|\mathcal{A}_{T}\right| \geqslant k_{0} K T .
$$

In order to get rid of the dependence on the initial data observe that, as a consequence, $W(T) \geqslant 1$ for all $T \geqslant t_{0}$ (the time $t_{0}$ does depend on the initial data). Hence, the second inequality in 677 yields

$$
W(T) \geqslant K \int_{0}^{T} \min (1, W(t)) \mathrm{d} t \geqslant K\left(T-t_{0}\right) .
$$

This finishes the proof of Proposition 5.2

Proof of Theorem 5.1] Theorem 5.1 is an easy consequence of Proposition 5.2 and its proof. Let us start with the inequality (64),

$$
\frac{\mathrm{d}}{\mathrm{d} t} \int\left(u-u^{2} / 2\right) \mathrm{d} x \geqslant M \int u_{x}^{2} \mathrm{~d} x+M \int u(1-u)^{2} \mathrm{~d} x .
$$

Consider the set $B \subset[T, 2 T]$ of times $t \in[T, 2 T]$ such that

$$
M \int u(t, x)(1-u(t, x))^{2} \mathrm{~d} x \geqslant \varepsilon \int\left(u(t, x)-u^{2}(t, x) / 2\right) \mathrm{d} x .
$$

Let us set

$$
Q(t)=\int\left(u(t, x)-u^{2}(t, x) / 2\right) \mathrm{d} x
$$

Exactly as in the proof of Proposition 5.2 we deduce that

$$
C_{1}\left(t-t_{0}\right) \leqslant Q(t) \leqslant C_{2}\left(t_{0}+t\right) .
$$

As $Q(t)$ is increasing in time, integrating 6 (69) over $B$ we obtain

$$
Q(2 T) \geqslant Q(T) e^{\varepsilon|B|} .
$$

For $T>10 t_{0}$ it follows that

$$
4 C_{2} T \geqslant C_{1} T e^{\varepsilon|B|},
$$

so that $|B| \leqslant K / \varepsilon$ with the constant $K$ independent of $T>t_{0}$. On the other hand, for $t \in[T, 2 T] \cap$ $B^{c}$ we have

$$
\varepsilon^{p}\left|\left\{x: u(t, x)|1-u(t, x)|^{2} \geqslant \varepsilon^{p}\right\}\right| \leqslant C \int u(t, x)(1-u(t, x))^{2} \mathrm{~d} x \leqslant C \varepsilon \int u(t, x) \mathrm{d} x,
$$

and 61) follows. 
5.2 Nonlinear asymptotic stability of the homogeneous state $(1,1)$

In this section, we consider the Keller-Segel-Fisher system and we consider the stability of the state $(1,1)$ as discussed in the introduction (see (14)-(15)). Therefore, we set $u=1+U$ and $v=1+V$ and the system 60 reads

$$
\left\{\begin{array}{l}
U_{t}-U_{x x}+\chi\left(V_{x} U\right)_{x}=-U(1+U)-\chi V_{x x}, \\
-d V_{x x}+V=U, \\
U(t=0, x)=U_{0}(x):=u^{0}-1, \quad x \in \mathbb{R} .
\end{array}\right.
$$

We prove that the linear stability of the homogeneous equilibrium state $(u=1, v=1)$ implies its nonlinear asymptotic stability. More precisely

THEOREM 5.3 For $\chi<(1+\sqrt{d})^{2}$, there is a positive constant $\delta>0$ such that for any initial data $u_{0}=1+U_{0}$ with $\int_{\mathbb{R}} U_{0}^{2}<\delta$, the solution $u$ of the Cauchy problem 60 converges to 1 in the $L^{2}$ norm with an exponential rate:

$$
\int_{\mathbb{R}}(u(t, x)-1)^{2} \mathrm{~d} x \rightarrow 0 \quad \text { as } t \rightarrow+\infty .
$$

Proof of Theorem 5.3. For $(t, x) \in \mathbb{R}^{+} \times \mathbb{R}$, we set $U(t, x):=u(t, x)-1, V(t, x):=v(t, x)-1$ and $\lambda:=\left(1+\sqrt{d)^{2}}-\chi>0\right.$. Multiplying equation 71 by $U$ and integrating over $\mathbb{R}$, we find

$$
\begin{aligned}
\frac{1}{2} \frac{\mathrm{d}}{\mathrm{d} t} \int_{\mathbb{R}} U^{2} \mathrm{~d} x & +\int_{\mathbb{R}}\left(U_{x}^{2}-\chi U_{x} V_{x}+U^{2}\right) \\
& =-\int_{\mathbb{R}} U^{3}+\chi \int_{\mathbb{R}} U U_{x} V_{x}=-\int_{\mathbb{R}} U^{3}-\frac{\chi}{2} \int_{\mathbb{R}} U^{2} V_{x x} \\
& =\left(\frac{\chi}{2 d}-1\right) \int U^{3}-\frac{\chi}{2 d} \int_{\mathbb{R}} U^{2} V \leqslant\left|\frac{\chi}{2 d}-1\right| \int|U|^{3}+\frac{\chi}{2 d} \int_{\mathbb{R}} U^{2}|V| .
\end{aligned}
$$

The second term on the left side of (73) can be written as

$$
\begin{aligned}
\int_{\mathbb{R}}\left(U_{x}^{2}-\chi U_{x} V_{x}+U^{2}\right) \mathrm{d} x & =\int_{\mathbb{R}}\left(\xi^{2}+1-\frac{\chi \xi^{2}}{1+\mathrm{d} \xi^{2}}\right)|\hat{U}(\xi)|^{2} \mathrm{~d} \xi \\
& =\int_{\mathbb{R}} \frac{P(\xi)}{\left(1+\mathrm{d} \xi^{2}\right)\left(1+\xi^{2}\right)}\left(1+\xi^{2}\right)|\hat{U}(\xi)|^{2} \mathrm{~d} \xi
\end{aligned}
$$

where $P$ is a fourth order poynomial function which is positive since $\chi<(1+\sqrt{d})^{2}$.

As $p(\xi)=\left(1+\mathrm{d} \xi^{2}\right)\left(1+\xi^{2}\right)$ is also a positive fourth order polynomial function, the quotient $P(\xi) /\left[\left(1+\mathrm{d} \xi^{2}\right)\left(1+\xi^{2}\right)\right]$ has a positive infimum $\lambda>0$. This gives

$$
\int_{\mathbb{R}}\left[\left|U_{x}\right|^{2}-\chi U_{x} V_{x}+U^{2}\right] \mathrm{d} x \geqslant \lambda \int_{\mathbb{R}}\left(1+\xi^{2}\right)|\hat{U}(\xi)|^{2} \mathrm{~d} \xi \geqslant \lambda \int_{\mathbb{R}}\left(U_{x}^{2}+U^{2}\right) \mathrm{d} x .
$$

Next, set $I(t)=\int_{\mathbb{R}} U^{2} \mathrm{~d} x$. The above computation yields

$$
\frac{1}{2} \frac{\mathrm{d}}{\mathrm{d} t} I(t)+\lambda I(t)+\lambda \int_{\mathbb{R}} U_{x}^{2} \leqslant\left|\frac{\chi}{2 d}-1\right| \int|U|^{3}+\frac{\chi}{2 d} \int_{\mathbb{R}} U^{2}|V| .
$$


We treat the two terms of the right side separately using Gagliardo-Nirenberg-Sobolev type inequalities:

$$
\int_{\mathbb{R}}|U|^{3} \leqslant C\left(\int_{\mathbb{R}} U_{x}^{2}\right)^{1 / 4}\left(\int_{\mathbb{R}} U^{2}\right)^{5 / 4} \leqslant \frac{\lambda}{2|\chi /(2 d)-1|} \int_{\mathbb{R}} U_{x}^{2}+M\left(\int_{\mathbb{R}} U^{2}\right)^{5 / 3}
$$

(the second inequality follows from the Minkowski inequality). In the same way we obtain

$$
\begin{aligned}
\int_{\mathbb{R}}|U|^{2}|V| & \leqslant\left(\int_{\mathbb{R}}|U|^{4} \int_{\mathbb{R}} V^{2}\right)^{1 / 2} \leqslant C_{1}\left(\int_{\mathbb{R}} U_{x}^{2}\right)^{1 / 4}\left(\int_{\mathbb{R}} U^{2}\right)^{3 / 4}\left(\int_{\mathbb{R}} U^{2}\right)^{1 / 2} \\
& \leqslant \frac{\lambda d}{\chi} \int_{\mathbb{R}} U_{x}^{2}+M^{\prime}\left(\int_{\mathbb{R}} U^{2}\right)^{5 / 3}
\end{aligned}
$$

where $M^{\prime}$ is a constant that only depends on $C_{1}, \chi, d$ and $\lambda$. This finally gives

$$
\frac{1}{2} \frac{\mathrm{d}}{\mathrm{d} t} I(t)+\lambda I(t)+\lambda \int_{\mathbb{R}} U_{x}^{2} \leqslant \lambda \int_{\mathbb{R}} U_{x}^{2}+\left(M+M^{\prime}\right) I^{5 / 3}(t),
$$

and thus for some constant $M^{\prime \prime}$ we have

$$
\frac{1}{2} \frac{\mathrm{d}}{\mathrm{d} t} I(t)+\lambda I(t) \leqslant M^{\prime \prime} I^{5 / 3}(t)
$$

Set now $\delta=\left(\lambda / M^{\prime \prime}\right)^{3 / 2}$. Then, for $I(0)<\delta$, the differential inequality 78 implies that $t \mapsto$ $I(t)$ decreases. As it is a nonnegative function, it converges to the equilibrium state $I \equiv 0$. Also, there is an exponential decay (with rate as close to $2 \lambda$ as we wish), and the proof is complete.

\section{Acknowledgements}

The work of LR was supported by NSF grant DMS-0604687 and that of BP by ANR project 05BLAN-0138. This work was done during visits by BP to the University of Chicago and by LR to ENS, Paris. We thank these institutions for their hospitality. The authors also wish to thank an anonymous referee whose remarks were useful to improve this paper.

\section{REFERENCES}

1. Ben-JaCob, E., Cohen, I., Golding, I., \& Kozlovsky, Y. Modeling branching and chiral colonial patterning of lubricating bacteria. Mathematical Models for Biological Pattern Formation, IMA Vol. Math. Appl. 121, Springer, New York (2001), 211-253. Zbl 1026.92003 MR 1796119

2. Berestycki, H., Constantin, P., \& Ryzhik, L. Non-planar fronts in Boussinesq reactive flows. Ann. Inst. H. Poincaré Anal. Non Linéaire 23 (2006), 407-437. Zbl pre05060812 MR 2245751

3. Berestycki, H., Nicolaenko, B., \& Scheurer, B. Traveling wave solutions to combustion models and their singular limits. SIAM J. Math. Anal. 16 (1985), 1207-1242. Zbl 0596.76096 MR 0807905

4. BRITtOn, N. F. Spatial structures and periodic travelling waves in an integro-differential reactiondiffusion population model. SIAM J. Appl. Math. 50 (1990), 1663-1688. Zbl 0723.92019 MR 1080515

5. CAlvez, V., \& CARrillo, J. A. Volume effects in the Keller-Segel model: energy estimates preventing blow-up. J. Math. Pures Appl. (9) 86 (2006), 155-175. Zbl 1116.35057 $\mid$ MR 2247456 
6. Constantin, P., Lewicka, M., \& RyzhiK, L. Travelling waves in two-dimensional reactive Boussinesq systems with no-slip boundary conditions. Nonlinearity 19 (2006), 2605-2615. Zbl 1106.76076 MR 2267720

7. Corrias, L., Perthame, B., \& ZaAg, H. Global solutions of some chemotaxis and angiogenesis systems in high space dimensions. Milan J. Math. 72 (2004), 1-28. Zbl 1115.35136 MR 2099126

8. Coville, J., \& Dupaigne, L. On a nonlocal equation arising in population dynamics. Proc. Roy. Soc. Edinburgh Sect. A 137 (2007), 727-755. Zbl 1133.35056 MR 2345778

9. Funaki, M., Mimura, M., \& Tsujikawa, T. Travelling front solutions arising in the chemotaxisgrowth model. Interfaces Free Bound. 8 (2006), 223-245. Zbl 1106.35119 MR 2256842

10. Furter, J., \& GRINFeld, M. Local vs. non-local interactions in population dynamics. J. Math. Biol. 27 (1989), 65-80. Zbl 0714.92012 MR 0984226

11. Golding, I., Kozlovsky, Y., Cohen, I., \& Ben-JAcob, E. Studies of bacterial branching growth using reaction-diffusion models for colonial development. Phys. A 260 (1998), 510-554.

12. Gourley, S. A. Travelling front solutions for a nonlocal Fisher equation. J. Math. Biol. 41 (2000), 272-284. Zbl 0982.92028 MR 1792677

13. Horstmann, D. From 1970 until present: the Keller-Segel model in chemotaxis and its consequences. II. Jahresber. Deutsch. Math.-Verein. 106 (2004), 51-69. Z Zbl 1072.35007| MR 2073515

14. Horstmann, D., \& Stevens, A. A constructive approach to traveling waves in chemotaxis. J. Nonlinear Sci. 14 (2004), 1-25. Zbl 1063.35071 MR 2032519

15. KANEL', YA. I. Stabilization of solutions of the Cauchy problem for equations encountered in combustion theory. Mat. Sb. (N.S.) 59 (101) (1962), 245-288. MR 0157130

16. LEwickA, M. Existence of traveling waves in the Stokes-Boussinesq system for reactive flow. J. Differential Equations 237 (2007), 343-371. Zbl pre05166844 MR 2330951

17. MAINI, P. K. Applications of mathematical modelling to biological pattern formation. Coherent Structures in Complex Systems (Sitges, 2000), Lecture Notes in Phys. 567, Springer, Berlin (2001), 205217. Zbl 0985.92011 MR 1995108

18. Marchant, B. P., Norbury, J., \& Sherratt, J. A. Travelling wave solutions to a haptotaxisdominated model of malignant invasion. Nonlinearity 14 (2001), 1653-1671. Zbl 0985.92012 MR 1867097

19. Murray, J. D. Mathematical Biology. II. 3rd ed., Interdisciplinary Appl. Math. 18, Springer, New York (2003). Zbl 1006.92002 MR 1952568

20. Perthame, B. Transport Equations in Biology. Birkhäuser, Basel (2007). Zbl pre05081225 MR 2270822

21. Senba, T., \& Suzuki, T. Applied Analysis. Imperial College Press, London (2004). Zbl 1053.00001 MR 2093755

22. Volpert, A. I., Volpert, V. A., \& Volpert, V. A. Traveling Wave Solutions of Parabolic Systems. Transl. Math. Monogr. 140, Amer. Math. Soc., Providence, RI (1994). MR 1297766

23. XIN, J. Front propagation in heterogeneous media. SIAM Rev. 42 (2000), 161-230. Zbl 0951.35060 MR 1778352 